\title{
HISTOCHEMISTRY OF FIBRES OF MASSETER AND TEMPORALIS MUSCLES OF EDENTULOUS MONKEYS MACACA MULATTA
}

\author{
L. C. Maxwell ${ }^{1,5}$, J. A. MCNamara JR $\mathbf{R}^{2,4}$, D. S. Carlson ${ }^{2,3,4}$ and J. A. Faulkner ${ }^{1}$ \\ Departments of Physiology ${ }^{1}$, Anatomy ${ }^{2}$ and Anthropology ${ }^{3}$; and \\ the Center for Human Growth and Development, ${ }^{4}$ \\ University of Michigan, Ann Arbor, MI 48109, U.S.A.
}

\begin{abstract}
Summary-Muscle fibres of the masseter and temporalis muscles of female rhesus monkeys which had been edentulous for $4.5 \mathrm{yr}$ were analysed histochemically. Fibres were classified on the basis of activities of succinic acid dehydrogenase and myofibrillar adenosine triphosphatase. Capillaries were visualized by phosphatase activity. Succinate oxidase activity was determined by differential respirometry of aliquots of whole muscle homogenates. These data were compared to data obtained from the muscles of control adult female animals. Relative to control values, the muscles of edentulous animals had lower oxidative capacity, more fast fatigable (FF) and fewer fast fatigue resistant (FR) and slow fatigue resistant (S) fibres, smaller $S$ fibres and slightly reduced capillarity. These data indicate relative disuse of elevator muscle following removal of teeth.
\end{abstract}

\section{INTRODUCTION}

Skeletal muscles adapt both structurally and functionally to increased (Maxwell, Faulkner and Lieberman, 1973) and decreased (Faulkner, Maxwell and Lieberman, 1972) activity. The muscles of mastication are active both for postural support of the mandible (McNamara, 1974a) and during ingestion, mastication (McNamara, 1974b) and deglutition (McNamara and Moyers, 1973) of food. However, the edentulous condition and the use of dentures alter the functions of these muscles (Tallgren, 1961, 1963; Matthews and Yemm, 1973). Recent studies of the histochemical profiles and cross-sectional area of temporalis muscle fibres in denture wearers indicate that differences in functional activity may lead to morphological and physiological adaptations (Ringquist, 1974ab).

There have been no previous studies of the adaptive responses of the masseter and temporalis muscles following complete tooth removal in the absence of prosthetic replacement. We determined the histochemical characteristics, fibre cross-sectional area, capillarity and oxidative capacity of the masseter and temporalis muscles from rhesus monkeys which were edentulous for $4 \frac{1}{2}$ yrs to understand better the neuromuscular and skeletal changes in edentulous individuals.

\section{MATERIALS AND METHODS}

A total of 11 adult female rhesus monkeys (Macaca mulatta) were used. Seven already studied for age and sex variation in the muscles of mastication (Maxwell et al., 1979) were used as controls. Complete dental

${ }^{5}$ Present Address: Department of Physiology, University of Texas Health Science Center at San Antonio, 7703 Floyd Curl Drive, San Antonio, TX 78284, U.S.A. extractions were performed on 4 monkeys $4.5 \mathrm{yrs}$ before death (Jaul et al., 1979). The monkeys were fed fresh fruit and monkey chow (Purina) ad libitum throughout.

Before killing, each monkey was anaesthetized with ketamine $(10 \mathrm{mg} / \mathrm{kg} \mathrm{IM})$ followed by an intravenous injection of pentobarbital sodium. The right superficial masseter and the right temporalis muscles were exposed and samples were excised from standardized sites (Maxwell et al., 1979) near the anterior and posterior borders of the muscles. Samples taken from temporalis muscles were divided; one portion for histochemical analysis was quick frozen in isopentane cooled by dry ice and the other, for biochemical analysis, was placed in ice-cold physiological salt solution. Because of the small mass of masseter muscle in the edentulous animals, it was not possible to obtain both anterior and posterior samples for biochemical assays; instead, a sample for the homogenate was excised from medial to the anterior and posterior histochemical samples. In control animals, succinate oxidase data from this medial site were no different from that of the anterior site (Table 2).

Cross-sections $14 \mu \mathrm{m}$ thick were cut from the frozen blocks on a cryostat at $-20^{\circ} \mathrm{C}$. Sections were incubated for succinic acid dehydrogenase (SDH) (Nachlas et al., 1957), myofibrillar ATPase (Chayen, Bitensky and Butcher, 1973) and capillary membrane phosphatase (Maxwell et al., 1977) activities. A section of each muscle sample incubatcd for myofibrillar ATPase activity was projected at $\times 1000$ magnification and the outlines of $30-40$ individual fibres in each of 3 or $425 \times 40 \mathrm{~cm}$ sample areas were traced. At least 100 fibres were traced from each muscle. Fibres completely within the sample area and fibres which crossed the top and right boundaries and the top corners of the sample area were included in the sample. 
Table 1. Mean fibre cross-sectional area $\left(\mu \mathrm{m}^{2}\right)$ and percentage composition of masseter and temporalis muscles of control and edentulous monkeys. Data are mean \pm standard error of the mean

\begin{tabular}{|c|c|c|c|c|c|c|}
\hline & \multicolumn{3}{|c|}{ Controls } & \multicolumn{3}{|c|}{ Edentulous } \\
\hline & $\mathrm{S}$ & FR & $\mathrm{FF}$ & $\mathrm{S}$ & FR & FF \\
\hline \multicolumn{7}{|c|}{ Mean fibre area $\left(\mu \mathrm{m}^{2}\right)$} \\
\hline Anterior masseter & $2330 \pm 138$ & $1105 \pm 299$ & $1585 \pm 225$ & $1085 \pm 213^{*}$ & $921 \pm 310$ & $1873 \pm 149$ \\
\hline Posterior masseter & $2240 \pm 163$ & $1898 \pm 189$ & $2137 \pm 264$ & $791 \pm 419^{*}$ & $1520 \pm 211$ & $2050 \pm 377$ \\
\hline Anterior temporalis & $2242 \pm 230$ & $1617 \pm 178$ & $1678 \pm 166$ & $714 \pm 294^{*}$ & $1238 \pm 119$ & $1352 \pm 59$ \\
\hline \multicolumn{6}{|c|}{ Percentage composition } & $1398 \pm 219$ \\
\hline Anterior masseter & $92.14 \pm 3.50$ & $7.00 \pm 3.17$ & $0.29 \pm 0.18$ & $55.40 \pm 18.13$ & $8.75 \pm 4.84$ & $35.58 \pm 19.83$ \\
\hline Posterior masseter & $40.14 \pm 0.87$ & $38.43 \pm 6.99$ & $21.57 \pm 4.03$ & $10.98 \pm 6.51^{*}$ & $11.27 \pm 2.08^{*}$ & $82.63 \pm 5.87^{*}$ \\
\hline Anterior temporalis & $43.95 \pm 8.02$ & $27.02 \pm 5.73$ & $23.00 \pm 8.81$ & $9.85 \pm 5.57^{*}$ & $11.75 \pm 6.28$ & $78.40 \pm 6.51^{*}$ \\
\hline Posterior temporalis & $9.43 \pm 2.39$ & $26.67 \pm 7.40$ & $62.82 \pm 6.30$ & $0.19 \pm 0.19^{*}$ & $4.60 \pm 2.85^{*}$ & $95.23 \pm 2.84^{*}$ \\
\hline
\end{tabular}

* Edentulous significantly different from control $(p<0.05)$.

No studies are available of the contractile properties of single motor units of masticatory muscles on which to verify the contraction velocity of the muscle fibres. Nevertheless, we have classified muscle fibres on the basis of myofibrillar ATPase activity as fast or slow based on the work of Burke et al. (1971). The serial section incubated for SDH activity was projected, also at $\times 1000$ magnification, and the sample fibres were classified as fatigue resistant or fatigable (Burke et al., 1971). As succinate is oxidized, nitroblue tetrazolium accepts electrons forming diformazan, a blue precipitate. Fibres classified as fatigue resistant had distinct SDH activity and subsarcolemmal aggregates of diformazan, especially near capillaries. Fibres which were classified as fatigable had very little SDH activity and no sub-sarcolemmal aggregates of diformazan. Slow (S), fast-fatigue resistant (FR) and fastfatigable (FF) fibres were identified. A third serial section incubated for capillary membrane phosphatase was projected and the location of capillaries relative to sample fibres drawn on the tracings. The number of capillaries within the sample area as well as the number of capillaries adjacent to each fibre in the sample were counted. Fibre area was determined by planimetry. Mean fibre area, percentage composition (the percentage of each type of fibre in the sample), fibres per $\mathrm{mm}^{2}$, capillaries per $\mathrm{mm}^{2}$ and capillary to fibre ratio were calculated.

The muscle sample for biochemical assays was blotted, weighed and homogenized in 19 volumes of icecold $0.01 \mathrm{M}$ phosphate buffer ( $\mathrm{pH} 7.4)$. Succinate oxidase activity of aliquots of the homogenate was deter- mined by the method of Potter (1964) using a Gilson differential respirometer. Protein content of the homogenates was determined by the procedure of Lowry (Lowry et al., 1951), and activity was expressed as $\mu$ l of oxygen utilized per mg protein per min. Statistical measures were calculated for samples from the masseter muscle and from the anterior and posterior regions of the temporalis muscle of control and edentulous animals. Differences between the means were tested by $t$-test with significance accepted at the $p<0.05$ level. Unless otherwise specified, data are expressed as mean $\pm \mathrm{SEM}$.

\section{RESULTS}

Within masticatory muscles of control female monkeys, $\mathrm{S}$ fibres were larger than FR or FF fibres. In contrast, $\mathrm{S}$ fibres in the masticatory muscles of edentulous animals were smaller than FF fibres at each muscle site and smaller than FR fibres in the anterior masseter. This resulted from significantly smaller $\mathrm{S}$ fibres in the masseter and temporalis muscles of edentulous compared to control animals, with no difference in the size of FR or FF fibres (Table 1).

Compared to control animals, significantly fewer $\mathrm{S}$ fibres and more FF fibres were observed in the posterior masseter and in the anterior and posterior temporalis of the edentulous animals (Table 1). Compared to control animals, muscle samples from edentulous animals had lower succinate oxidase activity (Table 2), consistent with the differences in percentage

Table 2 . Succinate oxidase activity $\left(\mu 1 \mathrm{O}_{2} \cdot \mathrm{mg} \mathrm{protein}^{-1} \mathrm{~min}^{-1}\right)$ of homogenates of masseter and temporalis muscles of control and edentulous animals. Data are mean \pm standard error of the mean

\begin{tabular}{lcc}
\hline & Controls & Edentulous \\
\hline Anterior masseter & $0.4572 \pm 0.0227$ & $0.1224 \pm 0.0205^{*}$ \\
Posterior masseter & $0.4058 \pm 0.0295$ & \\
Anterior temporalis & $0.3298 \pm 0.0240$ & $0.1332 \pm 0.0516^{*}$ \\
Posterior temporalis & $0.1816 \pm 0.0242$ & $0.0925 \pm 0.0534^{*}$ \\
\hline
\end{tabular}

* Edentulous significantly different from control $(p<0.05)$. 


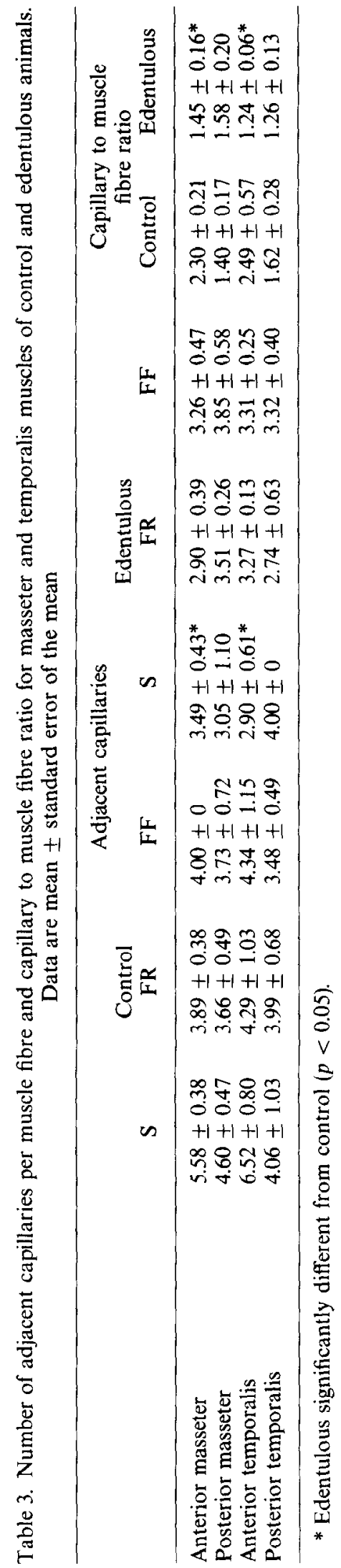

composition and in the intensity of SDH activity of individual muscle fibres (Fig. 1).

In anterior samples of both masseter and temporalis muscles, $\mathrm{S}$ fibres of edentulous animals had significantly fewer adjacent capillaries than $S$ fibres of control muscles (Table 3). No significant differences from control muscle were observed in the number of adjacent capillaries for other types of muscle fibres. This difference for $\mathrm{S}$ fibres resulted in a lower than control value for capillary to muscle fibre ratio of anterior masseter and anterior temporalis (Table 3).

\section{DISCUSSION}

Major adaptations take place within the masseter and temporalis muscles following removal of the dentition. Values for mean fibre area, proportion of fatigue-resistant fibres, oxidative capacity and capillarity of the masseter and temporalis muscles in the edentulous monkeys were all lower than in control animals. These data support the findings, yet to be published in full, of longitudinal EMG and cephalometric studies of the same experimental animals which showed alterations in functional activity and in the morphology of the maxilla and mandible. We found a reduction in the activity of the masseter muscle beginning immediately following tooth extractions and continuing for approximately six months to one year and Jaul et al. (1979) found reduction in the size of the mandible and of muscle force acting upon the mandible in edentulous animals.

Slow fibres were most affected by long-term absence of the dentition. Slow fibres are normally larger than fast fibres in the masseter and temporalis muscles of adult female rhesus monkeys (Maxwell et al., 1979) and human adults (Ringqvist, 1974a). Slow fibres are best adapted for sustained contractions of moderate force such as required for postural support and chewing. A smaller mean cross-sectional area of slow fibres probably indicates a decrease in the load resisted by these fibres. Reduction of load on slow fibres could result from an altered pattern of recruitment of these fibres due to a decreased role in maintenance of mandibular posture, decreased masticatory effort, or both. Slow fibres are probably recruited for both biting and mastication, and alteration of these functional activities in edentulous monkeys contributes to selective disuse atrophy of slow fibres.

Differences between control and edentulous monkeys in the proportion of each fibre type may also be accounted for by functional differences in muscle activity relating to maintenance of mandibular posture and mastication. Slow fibres are optimally suited for sustained contractions requiring relatively low force and, thus, are probably recruited during postural activity of the masseter and temporalis muscles. The reduced proportion of slow fibres in the masseter and temporalis muscles of edentulous monkeys relative to controls may result from a conversion of slow to fast fibres due to a sustained alteration in the pattern of recruitment of these fibres in response to altered jaw function. Conversion of slow fibres to fast fibres has been demonstrated in limb muscles in response to altered function (Booth and Kelso, 1973) and during growth (Maxwell et al., 1973; Kugelberg, 1976). 
Differences in the proportion and size of different fibre types between control and edentulous monkeys must be considered in terms of the variation which exists between normal adult male and female rhesus monkeys.

In masticatory muscles of normal adult female rhesus monkeys, fast fibres are smaller than slow fibres; however, the reverse is true among adult males, where fast fibres are larger than slow ones (Maxwell et al., 1979). This difference in relative fibre area probably correlates with sex differences in behaviour and mandibular morphology. As maximum bite force correlates with the mean area of fast fibres, but not with the mean area of slow fibres (Ringqvist et al., 1974a). the elevator musculature of female monkeys appears to be less adapted for forceful contractions than in the male. We infer that the fast fibres of adult females are not normally utilized for extremely forceful contractions, and thus show relatively little disuse atrophy following loss of the dentition. In adult males, complete tooth loss would likely result in atrophy of both fast and slow fibres.

The greater proportion of fast fatigable fibres in edentulous relative to control animals represents a loss of oxidative capacity of some fibres. Oxidative capacity of muscle fibres responds to the frequency with which the fibres are recruited during muscular activity, and changes in oxidative capacity of fibres occur easily. For example, greater oxidative capacity is observed in limb muscle fibres of endurance trained animals (Barnard, Edgerton and Peter, 1970; Maxwell et al., 1973), but is readily reversed by relative inactivity or de-training (Faulkner et al., 1972). Thus, reduced oxidative capacity of fibres of masticatory muscles of edentulous animals compared to control likely indicates relative disuse of these fibres. Reduced succinate oxidase activity in homogenates of masticatory muscles from edentulous compared to control monkeys adds further support to the detraining hypothesis.

The only difference in capillarity for the masseter and temporalis muscles of edentulous relative to control animals occurs adjacent to slow fibres. This results in a reduced capillary to fibre ratio for anterior samples of masseter and temporalis muscles of edentulous compared to control monkeys. Although the physiological significance of capillarity in anterior masseter and temporalis muscles is unclear, the possibility cannot be excluded that these differences may be related to greatly reduced activity by these muscles in edentulous animals. However, in control muscles of monkeys and other species, no relationship exists between capillary to muscle fibre ratio and either oxidative capacity or maximum muscle blood flow (Maxwell et al. 1977).

Tooth extraction in female rhesus monkeys alters mandibular morphology and muscle function, which results in adaptations in fibre area. percentage composition and oxidative capacity of masseter and temporalis muscles. These adaptations support our electromyographic data (unpublished) that the masseter and temporalis muscles are less active during postural support and during mastication in edentulous than in control animals. The predominance of adaptation occurs in the slow fibre population suggesting a response to a decreased role in mandibular support and to a decrease in the grinding of food by edentulous animals.

Acknowledgements - This research was supported by United States Public Health Service Grant DE-04227 and DE-05232, and by Contract DE-52478 from the National Institute of Dental Research, National Institutes of Health.

\section{REFERENCES}

Barnard R. J., Edgerton V. R. and Peter J. B. 1970. Effect of exercise on skeletal muscle. Biochemical and histochemical properties. J. appl. Physiol. 28, 762-766.

Booth F. W. and Kelso J. R. 1973. Effect of immobilization on contractile and histochemical properties of skeletal muscle. Pflugers Arch. 342, 231-238.

Burke R. E., Levine D. N., Zajac F. B., Tsairis P. and Engel W. K. 1971. Mammalian motor units: physiologicalhistochemical correlates of three types in cat gastrocnemius. Science, N.Y. 174, 709-712.

Chayen J., Bitensky L. and Butcher R. G. 1973. Practical Histochemistry, pp. 122-124. Wiley, New York.

Faulkner J. A., Maxwell L. C. and Lieberman D. A. 1972. Histochemical characteristics of muscle fibers from trained and detrained guinea pigs. Am. J. Physiol. 222, $836-840$.

Jaul D. H., McNamara Jr., J. A., Carlson D. S. and Upton L. G. 1979. A cephalometric evaluation of edentulous rhesus monkeys (Macaca mulatia): a long term study. $J$. prosthet. Dent. In press.

Kugelberg E. 1976. Adaptive transformation of rat soleus motor units during growth. J. neurol. Sci. 27, 269-289.

Lowry O. H., Rosebrough W. J., Farr A. L. and Randall R. S. 1951. Protein measurement with the Folin phenol reagent. J. biol. Chem. 193, 265-275.

Matthews B. and Yemm R. 1973. A silent period in the masseter electromyogram following tooth contact in subjects wearing full dentures. Archs oral Biol. 18, $531-535$.

Maxwell I. C. Carlson D. S., McNamara, Jr. J. A and Faukner J. A. 1979. Histochemical characteristics of the masseter and temporalis muscles of the rhesus monkey (Macaca mulatta). Anat. Rec. 193, 389-401.

Maxwell L. C.. Barclay J. K.. Mohrman D. E. and Faulkner J. A. 1977. Physiological characteristics of skeletal muscles of dogs and cats, Am. J. Physiol, 233, $\mathrm{C} 14 \mathrm{C} 18$.

Maxwell L. C., Faulkner J. A. and Lieberman D. A. 1973. Histochemical manifestations of age and endurance training in skeletal muscle fibers. Am. J. Physiol. 224, $356-361$.

McNamara J. A., Jr. 1974a. The electromyography of the mandibular postural position in the rhesus monkey (Macaca mulatta). J. dent. Res. 53, 949 .

McNamara J. A., Jr. 1974b. An electromyographic study of mastication in the rhesus monkey (Macaca mulatta). Archs oral Biol. 19, 821-823.

McNamara J. A., Jr. and Moyers R. E. 1973. Electromyography of the oral phase of deglutition in the rhesus monkey (Macaca mulatta). Archs oral Biol. 18, 9951002.

Nachlas M., Tsou M. K., DeSouza E., Cheng C. and Seligman A. M. 1957. Cytochemical demonstration of succinic dehydrogenase by the use of a new p-nitrophenyl substituted ditetrazole. J. Histochem. Cytochem. 5, $420-436$.

Potter V. R. 1964. The homogenate technique. In: Manometric Techniques (Edited by Umbreit W. W., Burris $\mathbf{R}$ H. and Stauffer T. F.) pp. 159-176. Burgess, Minneapolis.

Ringqvist M. 1974a. Fiber types in human masticatory muscles. Relation to function. Scand. $J$. dent. Res. 82 , $333-355$. 
Ringqvist M. 1974b. A histochemical study of temporal muscle fibers in denture wearers and subjects with normal dentition. Scand. J. dent. Res. 82, 28-39.

Tallgren A. 1961. An electromyographic study of the response of certain facial and jaw muscles to loss of teeth and subsequent complete denture treatment. Scand. dent. J. 69, 385-430.

Tallgren A. 1963. An electromyographic study of the behavior of certain facial and jaw muscles in long-term complete denture wearers. Odontol. Tidskr. 71, 425-444.

Plate 1 overleaf. 
Plate 1.

Fig. 1. Photomicrographs of cross-sections of the anterior masseter muscle of control and experimental animals. Serial sections from a control animal incubated for SDH (A) and myofibrillar ATPase (B) activities and serial sections from an edentulous monkey incubated for SDH (C) and myofibrillar ATPase (D) activities. Representative S, FR and FF fibres are identified. $\times 200$ 


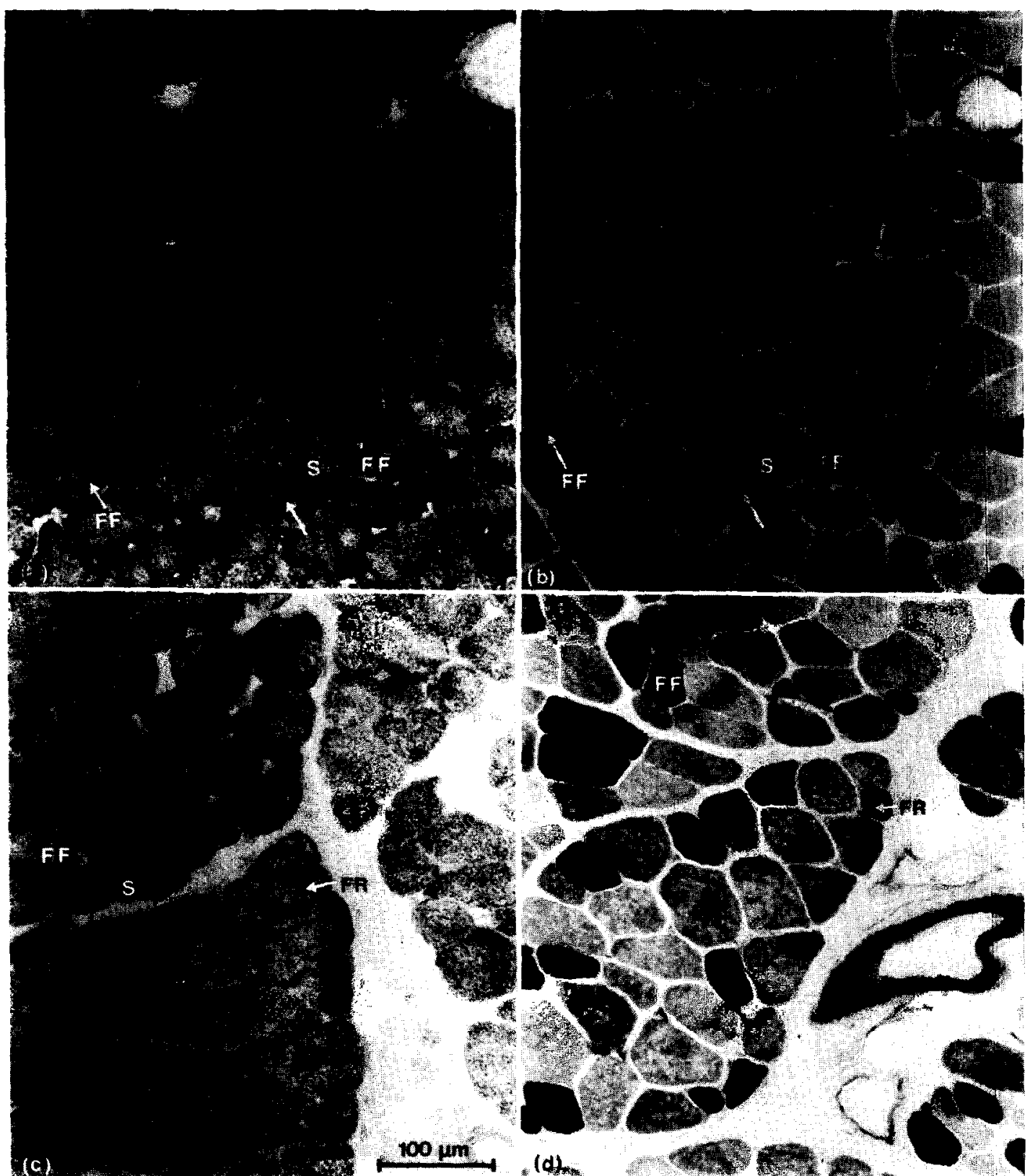
Plate I 